\title{
Synthesis and properties of PI polyamide-SAHA conjugate
}

\section{$\operatorname{AUTHOR}(\mathrm{S})$ :}

Ohtsuki, Akimichi; Kimura, Makoto T.; Minoshima, Masafumi; Suzuki, Tsukasa; Ikeda, Maki; Bando, Toshikazu; Nagase, Hiroki; Shinohara Ken-ichi; Sugiyama, Hiroshi

\section{CITATION:}

Ohtsuki, Akimichi ...[et al]. Synthesis and properties of PI polyamideSAHA conjugate. Tetrahedron Letters 2009, 50(52): 7288-7292

\section{ISSUE DATE:}

2009-12-30

URL:

http://hdl.handle.net/2433/88220

\section{RIGHT:}

c 2009 Elsevier Ltd. All rights reserved.; この論文は出版社版でありませ ん。引用の際には出版社版をご確認ご利用ください。; This is not the published version. Please cite only the published version. 


\section{Graphical Abstract}

To create your abstract, type over the instructions in the template box below. Fonts or abstract dimensions should not be

\section{Synthesis and Properties of PI polyamide-}

\section{SAHA conjugate}

Akimichi Ohtsuki ${ }^{\mathrm{a}}$, Makoto T. Kimura ${ }^{\mathrm{b}}$, Masafumi Minoshima ${ }^{\mathrm{a}}$, Tsukasa Suzuki ${ }^{b}$, Maki Ikeda ${ }^{b}$, Toshikazu Bando ${ }^{a}$, Hiroki Nagase $^{\mathrm{b}}$, Ken-ichi Shinohara ${ }^{\mathrm{a}}$, Hiroshi Sugiyama, ${ }^{\mathrm{a}, \mathrm{c}^{*}}$ ${ }^{a}$ Department of Chemistry, Graduate School of Science, Kyoto University, Kitashirakawa-Oiwakecho, Sakyo, Kyoto 606-8502, ' Japan, Division of Cancer Genetics, Department of Advanced Medical Science, Nihon University School of Medicine, Tokyo 173-8610, Japan 'Institute for Integrated Cell-Material Sciences, Kyoto University, Sakyo, Kyoto 606-8502, Japan

changed or altered. 


\title{
Synthesis and Properties of PI polyamide-SAHA conjugate
}

\author{
Akimichi Ohtsuki ${ }^{\mathrm{a}}$, Makoto T. Kimura ${ }^{\mathrm{b}}$, Masafumi Minoshima ${ }^{\mathrm{a}}$, Tsukasa Suzuki ${ }^{\mathrm{b}}$, Maki Ikeda ${ }^{\mathrm{b}}$, \\ Toshikazu Bando ${ }^{\mathrm{a}}$, Hiroki Nagase ${ }^{\mathrm{b}}$, Ken-ichi Shinohara ${ }^{\mathrm{a}}$, Hiroshi Sugiyama ${ }^{\mathrm{a}}$ \\ ${ }^{a}$ Department of Chemistry, Graduate School of Science, Kyoto University, Kitashirakawa-Oiwakecho, Sakyo, Kyoto 606-8502, Japan, \\ ${ }^{b}$ Division of Cancer Genetics, Department of Advanced Medical Science, Nihon University School of Medicine, Tokyo 173-8610, Japan, \\ ${ }^{c}$ Institute for Integrated Cell-Material Sciences, Kyoto University, Sakyo, Kyoto 606-8502, Japan, \\ * Corresponding author. Tel.: +81-75-753-4002; fax: +81-75-753-3670; e-mail: hs@kuchem.kyoto-u.ac.jp.
}

\begin{abstract}
We have designed and synthesized new types of pyrrole (P)-imidazole (I) polyamide conjugates 1 and 2 possessing a suberoylanilide hydroxamic acid (SAHA) moiety that is a strong inhibitor of histone deacetylase (HDAC). SAHA conjugate 2 was designed to target the promoter region of the p16 tumor suppressor gene. The DNA binding affinity of SAHA conjugate 2 to its target sequence was examined using surface plasmon resonance. HDAC inhibition activity of conjugates $\mathbf{1}$ and $\mathbf{2}$ was evaluated using a colorimetric assay. The results demonstrated that even though it possesses the relatively large SAHA moiety, conjugate 2 has high DNA sequence-specific binding properties and moderate HDAC inhibitory activity in vitro. SAHA conjugate 2 was found to cause morphological changes in HeLa cells and to induce selective Histone H3 lysine 9 acetylation. @ 2009 Elsevier eScienc. All rights reserved
\end{abstract}

Inhibitors of histone deacetylase (HDAC) have recently gained prominence as an emerging class of anticancer agents. ${ }^{1)}$ HDAC catalyzes the deacetylation of $\varepsilon$-amino groups of lysine residues in the $\mathrm{N}$-terminal tails of core histones in the nucleosome, resulting in protonated $\varepsilon$-amino lysine residues that interact strongly with DNA to give rise to an inactive compact chromatin structure. ${ }^{2)}$ In this way, HDAC can act as a regulator of gene transcription factors. Thus, inhibition of HDAC causes histone hyperacetylation, which leads to the disruption of chromatin structure and, as a result, the transcriptional activation of epigenetically silenced genes, such as tumor suppressor genes in cancer. ${ }^{3)}$ Indeed, HDAC inhibitors have been developed as anticancer agents. Suberoylanilide hydroxamic acid (SAHA, Figure 1) is a potential inhibitor of HDAC that chelates the $\mathrm{Zn}^{2+}$ cation at the HDAC active site to induce efficient inhibition. ${ }^{4}$ SAHA has recently been approved by the U.S. Food and Drug Administration (FDA) for the treatment of cutaneous T-cell lymphoma. Currently, there are over 50 clinical trials in progress at the National Institutes of Health (NIH, USA) relating to SAHA. ${ }^{5)}$ However, there is currently no system to deliver SAHA to a target region of the genome.<smiles>CC(/C=C/C=C/C(=O)NO)C(=O)c1ccc(N(C)C)cc1</smiles>

Figure 1. Chemical structures of suberoylanilide hydroxamic acid (SAHA) and trichostatin A (TSA).
To deliver SAHA to a target region of the genome, we used $N$-methylpyrrole (P) and $N$-methylimidazole (I) polyamides (PI polyamides). PI polyamides bind to the minor groove and recognize each of the four Watson-Crick base pairs of the double-stranded DNA in a sequencespecific manner. Antiparallel pairing of the I/P pair recognizes the $\mathrm{G} / \mathrm{C}$ base pair, whereas a $\mathrm{P} / \mathrm{P}$ pair recognizes $\mathrm{A} / \mathrm{T}$ or T/A base pairs. ${ }^{6)}$ Since the binding constants and specificity of PI polyamides are comparable to those of transcription factors, the expression of many genes was silenced by the binding of PI polyamides to their regulatory sequences. Various types of sequence-specific functional agents have been developed by conjugation of PI polyamides with peptides, alkylating agents and synthetic compounds. For example, upregulation of genes has been demonstrated by tethering of the PI polyamide to a dimerization element or activation domain. ${ }^{7,8)}$ There have been no reports of PI polyamide conjugates that can regulate histone modification. It is known that the p16 tumor suppressor gene is inactivated in many cancers. ${ }^{9)}$ In this paper, we report the synthesis and HDAC inhibitory activity of PI polyamide-SAHA conjugates $\mathbf{1}$ and $\mathbf{2}$ (Figure 2). SAHA conjugate 2 was designed to bind to the DNA sequence 5'-WGCWGGC-3', present in the promoter region of the tumor suppressor p16 gene. ${ }^{9)}$

The synthesis of PI polyamide-SAHA conjugates $\mathbf{1}$ and 2 is outlined in Figure 3. A six-ring PI polyamide $\mathbf{4}$ and tenring PI polyamide $\mathbf{6}$, with terminal amino groups, were prepared by Fmoc solid phase synthesis using a $\beta$-alaninecoupled CLEAR-acid resin. 4-(8-Methoxy-8oxooctanamido) benzoic acid 3 was prepared by coupling of 


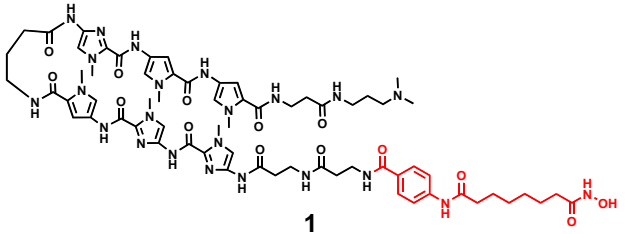

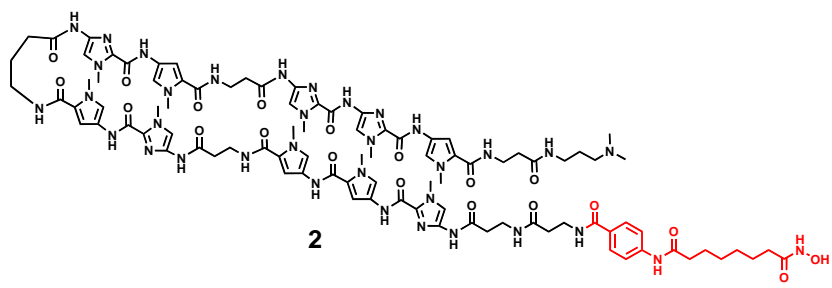

Figure 2. Chemical structures of PI polyamide-SAHA conjugates $\mathbf{1}$ and $\mathbf{2}$.
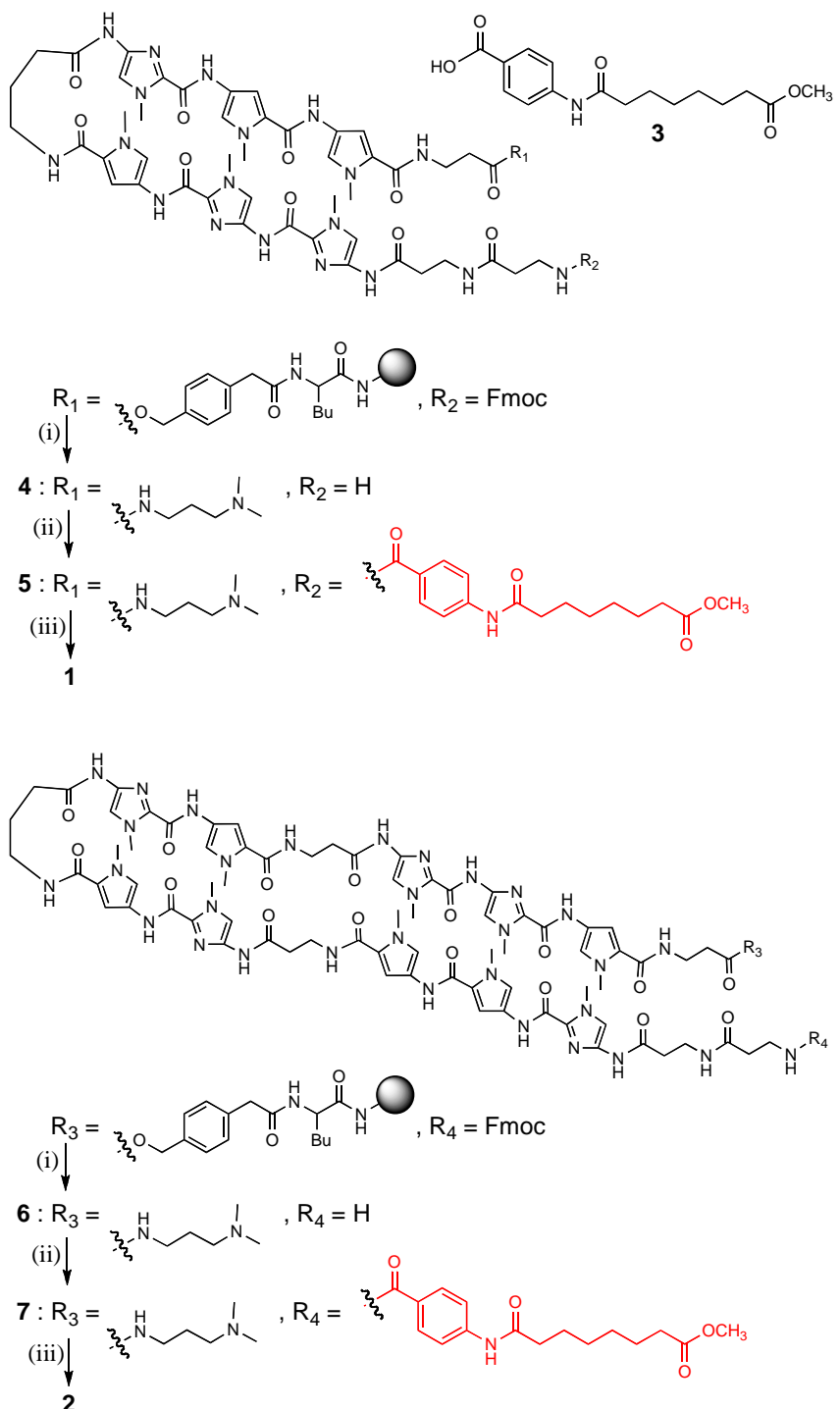

Figure 3. Synthetic scheme for the preparation of PI polyamide-SAHA conjugate 1 and 2. (i) $N, N$-Dimethyl-1,3-propanediamine, $55^{\circ} \mathrm{C}$, overnight. (ii) 4-(8-Methoxy-8-oxooctanamido)benzoic acid 3, FDPP, DIEA, DMF, room temperature, 6 hours. (iii) $\mathrm{NH}_{2} \mathrm{OH}, \mathrm{H}_{2} \mathrm{O}$, DMF, room temperature, 6 hours. amino benzoic acid with methyl 8-chloro-8-oxooctanoate, and converting to an activated ester using pentafluorophenyl diphenylphosphinate (FDPP) and $N, N$ diisopropylethylamine (DIEA), followed by coupling with PI polyamide $\mathbf{4}$ or $\mathbf{6}$ to produce 4-(8-methoxy-8oxooctanamido) benzoyl conjugated PI polyamide 5 or $\mathbf{7}$, respectively. ${ }^{10,11)}$ The conjugates $\mathbf{1}$ and $\mathbf{2}$ were prepared from PI polyamides 5 and 7 by amino-de-alkoxylation using a solution of hydroxylamine in water/dimethylformamide (DMF) (1/1). The conjugates 1 and 2 were confirmed by electrospray ionization time-of-flight (ESI-TOF) mass spectrometry after purification using reversed-phase HPLC. HDAC inhibition by purified conjugates $\mathbf{1}$ and $\mathbf{2}$ was evaluated in an in vitro assay.

Sequence-specific binding of PI polyamide-SAHA conjugate 2 was evaluated by surface plasmon resonance (SPR) experiments. 5'-Biotinylated hairpin DNA (oligonucleotide 1, ODN1) containing a single binding site (5'-GCWGGC-3') was prepared (as shown in Figure 4) and immobilized on a streptavidin-coated sensor chip. Dissociation equilibrium constants $(\mathrm{Kd})$ were obtained by fitting the resulting SPR sensorgrams (shown in Figures 5a and $b$ ) to a theoretical model based on previous studies. The $\mathrm{Kd}$ values for PI polyamide-SAHA conjugate $\mathbf{2}$ and its precursor polyamide $\mathbf{6}$ were determined as $3.79 \times 10^{-8} \mathrm{M}$ and $3.74 \times 10^{-8} \mathrm{M}$, respectively. These results demonstrated that the PI polyamide-SAHA conjugate has as high an affinity for matching DNA as PI polyamide with an $\mathrm{NH}_{2}$ terminal, and that the SAHA moiety does not inhibit binding of PI polyamide to DNA.

\section{5 ' - biotin - TTTGCTGGCAAAC T OO $\diamond 000 \diamond D p \quad T$} 3 ' - AAACGACCGTTTG T

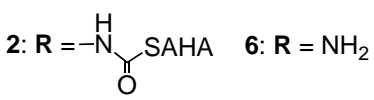

Figure 4. Schematic representation of the binding of PI polyamide-SAHA conjugate $\mathbf{2}$ and PI polyamide $\mathbf{6}$ to oligonucleotide 1 (ODN1). White circles represent pyrroles, black circles imidazoles, diamonds $\beta$-alanine and $D p$ is $\mathrm{N}, \mathrm{N}$-dimethylamino-3-propanediamine. The binding site is shown in red.

The inhibition of HDAC activity by PI polyamideSAHA conjugates $\mathbf{1}$ and $\mathbf{2}$ was examined by a colorimetric assay using an acetylated lysine derivative (Biomol International) following the manufacturer's protocol. HDAC inhibition was observed at $1000 \mathrm{nM}$ for the PI polyamideSAHA conjugates 1 and $\mathbf{2}$ shown in Figure 6. We confirmed that PI polyamide-SAHA conjugates 1 and $\mathbf{2}$ possess HDAC inhibiting activity, and that these activities were lower than that of TSA. In addition, the inhibitory activity of conjugate $\mathbf{2}$ was slightly lower than that of conjugate $\mathbf{1}$. These results suggest that the HDAC inhibitory activity of the SAHA moiety was negatively affected by the chain structures of PI polyamides. 
(a)

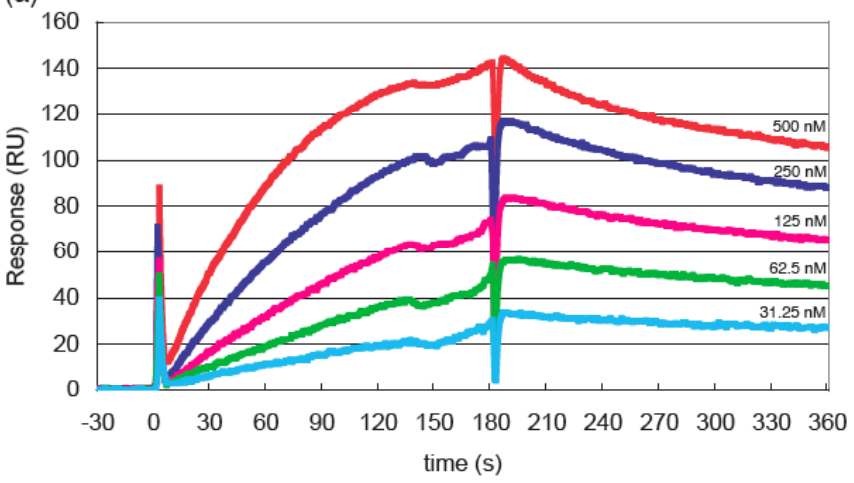

(b)

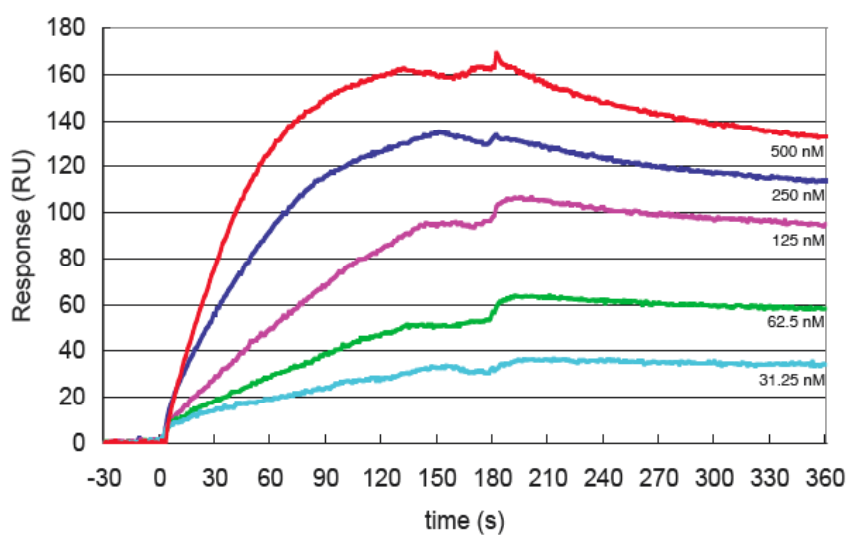

Figure 5. SPR sensorgrams for the interaction of PI polyamide SAHA conjugate 2 (a) and PI polyamide 6 (b) at a concentration of $31.25 \mathrm{nM}$ (cyan), $62.5 \mathrm{nM}$ (green), $125 \mathrm{nM}$ (magenta), $250 \mathrm{nM}$ (blue) and $500 \mathrm{nM}$ (red), with matching DNA (ODN1).

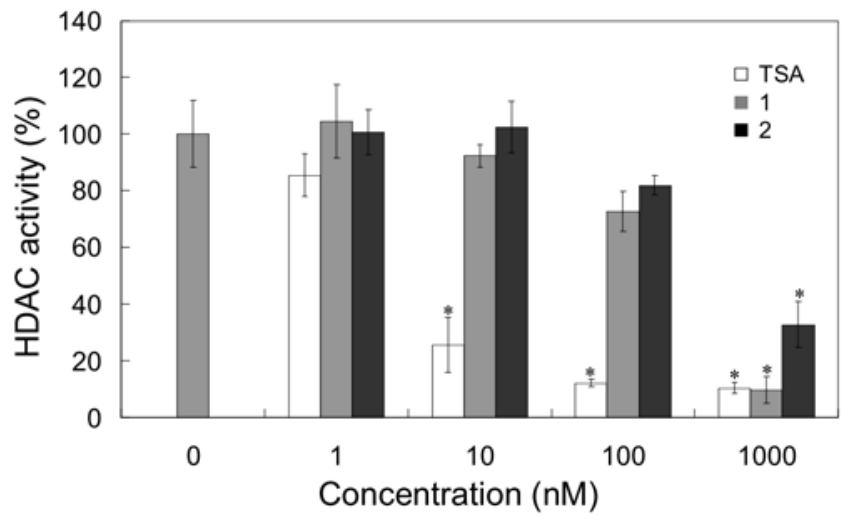

Figure 6. HDAC inhibition activities of TSA (white), conjugate $\mathbf{1}$ (gray), and $\mathbf{2}$ (black). The HDAC activities were determined fluorescently using a HDAC Colorimetric Assay/Drug Discovery kit (BIOMOL). Data represent the means of three independent experiments; error bars indicate SDs. *P < 0.05 by unpaired Student's t-test compared with no drug control $(0 \mathrm{nM})$.

To evaluate the biological impact of SAHA conjugates, HeLa cells were treated with $150 \mathrm{nM}$ SAHA conjugate 2, designed to bind to the 5'-WGCWGGC-3' sequence that exists in the promoter region of the p16 tumor suppressor gene. As shown in Figure 7, morphological changes of HeLa cells were observed after 2 weeks. HeLa cells treated with
SAHA conjugate 2 became swollen and had larger cytoplasms than cells treated with SAHA conjugate $\mathbf{1}$ whereas PI polyamide-SAHA conjugate 1, with its short recognition sequence, and PI polyamide $\mathbf{1}$, did not induce morphological changes of HeLa cells.
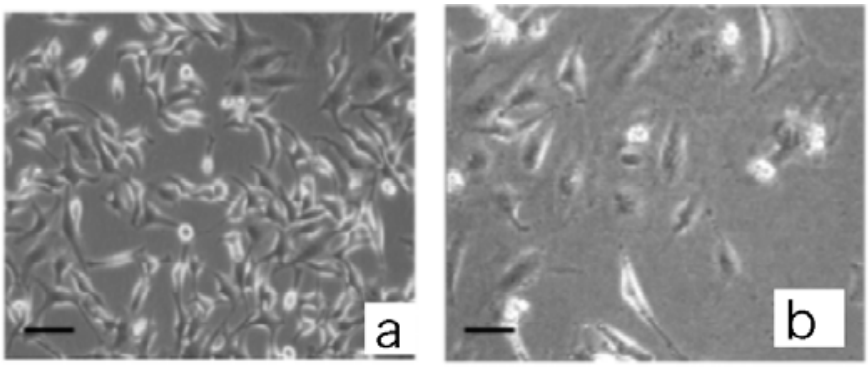

Figure 7. HeLa cells were cultured in complete Dulbecco's Modified Eagle's Medium (SIGMA, St. Louis, MO, USA) supplemented with $10 \%$ fetal bovine serum in $5 \% \mathrm{CO}_{2}$ at $37^{\circ} \mathrm{C}$ with following reagents: $150 \mathrm{nM}$ SAHA conjugate 1 (a) or conjugate 2 (b) for 2 weeks. Scale bar indicates $100 \mu \mathrm{m}$

To evaluate whether the SAHA conjugates affect histone acetylation of the p16 promoter region, we used a chromatin immunoprecipitation (ChIP) assay. The chromatins in HeLa cells treated with $150 \mathrm{nM}$ SAHA, SAHA conjugate 1 or SAHA conjugate 2 , were immunoprecipitated by antibodies against acetylated lysine 9 or complete histone H3. The coprecipitated DNAs were then amplified with the specific primer set for the p16 promoter sequence. The amount of DNA in the immunoprecipitants using each antibody was evaluated. As shown in Figure 8, histone H3 lysine 9 acetylation in the p16 promoter was twice as high in HeLa cells treated with SAHA conjugate 2 than SAHA conjugate 1. This result suggests that SAHA conjugate 2 has the ability to specifically induce acetylation at the target region of chromatin where PI polyamide binds. Conjugate $\mathbf{2}$ was designed to target the p16 promoter region. We therefore investigated whether conjugate 2 upregulated the expression level of p16 and induced G1 cell-cycle arrest in the treated cells. Unfortunately, the stable induction of p16 mRNA and protein during short-term treatment ( 7 days) was not observed. FACS analysis indicated that treatment with 150 $\mathrm{nM}$ of conjugate 2 for 7 days resulted in weak G1 arrest in cells. A similar effect was also seen after treatment with conjugate 2 (supplementary information). Since it is well known that SAHA itself induces G1 arrest in cancer cells and 2-day treatment with $150 \mathrm{nM}$ SAHA increases sub-G1 positive cells, this result might be because of the effect of the SAHA moiety. 


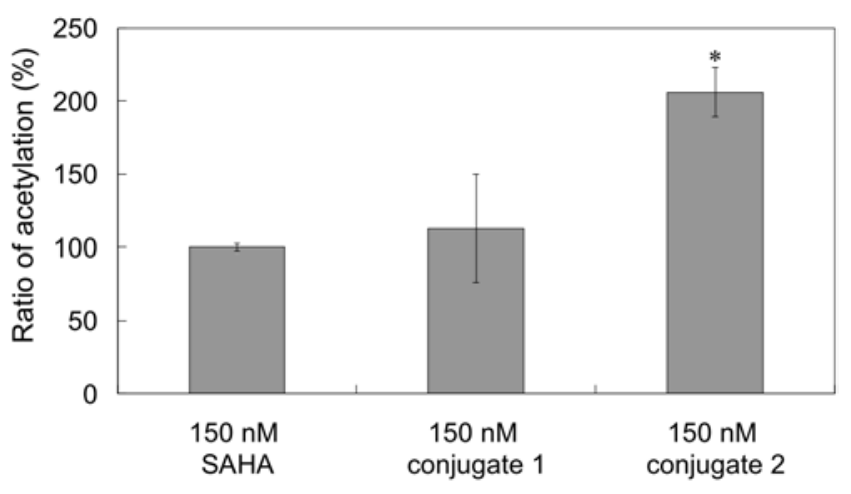

Figure 8. HeLa cells were treated with the indicated reagents (150 nM) for seven days. After immunoprecipitation by acetylated (lysine 9) or whole H3 antibody, the amount of p16 promoter sequence in the co-precipitated DNAs was determined by quantitative PCR. We set the ratio of the amount of PCR products in acetylated/whole immunoprecipitants in $150 \mathrm{nM}$ SAHA-treated cells as $100 \%$ in this graph. Data represent the means of three independent experiments; error bars indicate SDs. ${ }^{*} \mathrm{P}<0.05$ by unpaired Student's $t$ test compared with 150 nM SAHA.

In summary, we synthesized and evaluated PI polyamide-SAHA conjugates. PI polyamide-SAHA conjugate 2 has high DNA sequence-specific binding ability and HDAC inhibitory activity. These results suggest that PI polyamide-SAHA conjugates use the PI polyamide moiety as a carrier to a specific DNA sequence, where they induce acetylation of the chromatin. PI-polyamides with chromatin regulators, such as SAHA, are useful molecules to regulate chromatin modification at a specific region of the chromosome.

\section{Acknowledgments}

This research was supported in part by the Global COE program "International Center for Integrated Research and Advanced Education in Materials" (No. B-024) of the Ministry of Education, Culture, Sports, Science and Technology (MEXT) of Japan, administrated by the Japan Society for the Promotion of Science and the Academic Frontier 2006 from MEXT to HN.

\section{Supplementary data}

Supplementary data (experimental procedures and characterization of all new compounds) associated with this article can be found, in the online version, at doi:.

\section{References and notes}

1. Xu, W. S.; Parmigiani, R. B.; Marks, P. A. Oncogene, 2007, 26, $5541-5552$

2. (a) Wolffe, A. P. Science, 1996, 272, 371-372. (b) Butler, L. M.; Zhou, X.; Xu, W. S.; Scher, H. I.; Rifkind, R. A.; Marks, P. A; Richon, V. M. Proc. Natl. Acad. Sci. U.S.A. 2002, 99, 11700-11705. (c) Hassig, C. A.; Schreiber, S. L. Curr. Opin. Chem. Biol., 1997, 1, 300-308. (d) Lahery, C. D.; Yanh, W. M.; Sun, J. M.; Davie, J. R.; Seto, E.; Eisenman, R. N. Cell, 1997, 89, 349-356.

3. (a) Saito, A.; Yamashita, T.; Mariko Y.; Nosaka, Y.; Tsuchiya, K.; Ando, T. Proc. Natl. Acad. Sci. U.S.A. 1999, 96, 45924597. (b) Yoshida, M.; Kijima, M.; Akita, M.; Beppu, T. J. Biol. Chem., 1990, 265, 1714-1719.

4. Richon, V. M.; Emilian, S.; Verdin, E.; Webb, Y.; Breslow, R.; Rifkind, R. A.; Marks, P. A. Proc. Natl. Acad. Sci., 1998, 95, 3003-3007.

5. Garber, K.; Nature Biotechnology, 2007, 25, 17-19.

6. (a) White, S.; Szewczyk, J. W.; Turner, J. M.; Baird, E. E.; Dervan, P. B.; Edelson, B. S. Curr. Opin. Struct. Biol., 2003, 13, 284-299. (c) Dervan, P. B.; Poulin-Kerstein, A. T.; Fecher, E. J.; Edelson, B. S. Top. Curr. Chem., 2005, 253, 1-31.

7. (a) Wang, C. C. C.; Ellervik, U.; Dervan, P. B. Bioorg. Med. Chem., 2001, 9, 653-657. (b) Turner, J. M.; Swalley, S. E.; Baird, E. E.; Dervan, P. B. J. Am. Chem. Soc., 1998, 120, 6219-6226.

8. (a) Kwon, Y.; Arndt, H. D.; Mao, Q.; Choi, Y.; Kawazoe, Y.; Dervan, P. B.; Uesugi, M. J. Am. Chem. Soc., 2004, 126, 15940-15941. (b) Ansari, A. Z.; Mapp, A. K.; Nguyen, D. K.; Dervan, P. B.; Ptashne, M. Chemistry and Biology, 2001, 8, 583-592.

9. (a) Matheu, A.; Maraver, A.; Serrano, M. Cancer Res., 2008, 68, 6031-6034. (b) Ansari, A. Z.; Mapp, A. K.; Nguyen, D. K.; Dervan, P. B.; Ptashne, M. J. Exp. Clin. Cancer Res., 2007, 26, 443-449. (c) Matheu, A.; Maraver, A.; Serrano, M. Cancer Epidemiol. Biomarkers Prev., 2008, 17, 2174-2179. (d) Baylin, S. B.; Ohm, J. E. Nat. Rev. Cancer, 2006, 6, 107-116.

10. (a) Swalley, S. E.; Baird, E. E.; Dervan, P. B. J. Am. Chem. Soc., 1997, 119, 6953-6961. (b) Salisbury, C. M.; Cravatt, B. C.; Proc. Natl. Acad. Sci. U.S.A. 2007, 104, 1171-1176.

11. (a) Minoshima, M.; Bando, T.; Sasaki, S.; Fujimoto, J.; Sugiyama, H. Nucleic Acids Research, 2008, 36, 2889-2894. (b) Minoshima, M.; Bando, T.; Sasaki, S.; Shinohara, K.; Fujimoto, J.; Sugiyama, H. J. Am. Chem. Soc.; 2007, 129, 5384-5390. (c) Zhang, W.; Bando, T.; Sugiyama, H. J. Am. Chem. Soc.; 2006, 128, 8766-8776. 